
\title{
.
}

\section{Phosphatidylinositol Induces Caspase- Independent Apoptosis of Malignant Pleural Mesothelioma Cells by Accumulating AIF in the Nucleus}

\author{
Shingo Kanemura ${ }^{a}$ Ayako Tsuchiya ${ }^{b}$ Takeshi Kanno $^{b}$ Takashi Nakano ${ }^{a}$ \\ Tomoyuki Nishizaki ${ }^{b}$ \\ aDivision of Respiratory Medicine, Department of Internal Medicine and bivision of Bioinformation, \\ Department of Physiology, Hyogo College of Medicine, Nishinomiya, Japan
}

\section{Key Words}

Phosphatidylinositol - Malignant pleural mesothelioma - Caspase-independent apoptosis • Bid • AIF

\begin{abstract}
Background/Aims: Phosphatidylinositol (PI) regulates a variety of cell processes. The present study investigated the antitumor action of 1,2-dioleoyl-sn-glycero-3-phospho-(1'-myoinositol)(DOPI) and 1,2-dipalmitoyl-sn-glycero-3-phospho-(1'-myo-inositol)(DPPI) on human malignant pleural mesothelioma (MPM) cell lines such as NCI-H28, NCI-H2052, NCI-H2452, and MSTO-211H cells. Methods: MTT assay, TUNEL staining, flow cytometry using propidium iodide (PI) and annexin V (AV), enzymatic caspase assay, and nuclear staining using DAPI were carried out, and mitochondrial membrane potentials and intracellular distribution of apoptosisinducing factor (AIF) were monitored in cells with and without the siRNA silencing the Bidtargeted gene. Results: Both DOPI and DPPI reduced cell viability for all the investigated MPM cell lines in a concentration (0.01-100 $\mu \mathrm{M})$-dependent manner. DOPI and DPPI significantly increased TUNEL-positive cells and the population of PI-negative/AV-positive and PI-positive/ AV-positive cells, corresponding to early apoptosis and late apoptosis/secondary necrosis, respectively. DOPI and DPPI perturbed mitochondrial membrane potentials in MSTO- $211 \mathrm{H}$ cells, but no significant activation of caspase- $3,-4,-8$, and -9 was obtained. DOPI and DPPI upregulated expression of Bid in MSTO- $211 \mathrm{H}$ cells. DOPI and DPPI significantly increased nuclear localization of AIF without affecting expression of the mRNAs and proteins in MSTO-211H cells, which was inhibited by knocking-down Bid. In the DAPI staining, nuclear fragmentation and condensation were found. Conclusion: The results of the present study indicate that DOPI and DPPI facilitate Bid-mediated AIF release from the mitochondria, to accumulate AIF in the nucleus and induce caspase-independent apoptosis of MPM cells.
\end{abstract}




\section{Cellular Physiology Cell Physiol Biochem 2015;36:1037-1048 \begin{tabular}{l|l|l|} 
DOI: 10.1159/000430277 & (0) 2015 S. Karger AG, Basel
\end{tabular} and Biochemistry Published online: June 18, 2015 www.karger.com/cpb \\ Kanemura et al.: Phosphatidylinositol-Induced Apoptosis}

\section{Introduction}

Phosphatidylinositol (PI) is a member of plasma membrane components, and PI by itself and its metabolites are indispensable for a wide-variety of cellular responses. Phosphatidylinositol 4,5-bisphosphate ( $\mathrm{PIP}_{2}$ ) is hydrolyzed into diacylglycerol and inositol 1,4,5-trisphosphate $\left(\mathrm{IP}_{3}\right)$ by phospholipase $\mathrm{C}[1,2]$. $\mathrm{IP}_{3}$ releases $\mathrm{Ca}^{2+}$ through $\mathrm{IP}_{3}$-gated calcium channel on the endoplasmic reticulum, to activate conventional protein kinase $\mathrm{C}$ (PKC) isozymes together with diacylglycerol [1,2]. PI, alternatively, is a critical mediator in a receptor tyrosine kinase (RTK) signaling pathway. RTK phosphorylates its own receptor and insulin receptor substrate 1 (IRS-1), to recruit and activate phosphatidylinositol 3-kinase (PI3K), which phosphorylates $\mathrm{PIP}_{2}$ to produce phosphatidylinositol 3,4,5-trisphosphate $\left(\mathrm{PIP}_{3}\right) . \mathrm{PIP}_{3}$ binds to and activates 3-phosphoinositide-dependent protein kinase 1 (PDK1), which in turn, activates Akt followed by Rac1/Cdc42. Moreover, PI regulates cell proliferation, differentiation, migration, chemotaxis, phagocytosis, and survival [3]. PI is also involved in the regulation of vesicular trafficking, membrane dynamics, actin cytoskeleton organization, activation of ion channels, and transporters [4, 5]. Amazingly, PI has the potential to activate PKCs or inhibit protein phosphatases, particularly protein tyrosine phosphatase 1B (PTP1B) [6]. We have earlier found that the PI derivative 1,2-O-bis-[8-\{2-(2-pentylcyclopropylmethyl)-cyclopropyl\}-octanoyl]-sn-glycero-3-phosphatidyl-D-1-inositol (diDCPLA-PI) induces apoptosis of MKN28 human gastric cancer cells by suppressing activity of mitogen-activated protein kinase kinase (MEK)[7].

Apoptosis is induced in a caspase-dependent and -independent manner. Caspasedependent apoptosis is executed through oxidative stress-induced mitochondrial damage involving caspase-9 activation, endoplasmic reticulum (ER) stress involving caspase-4 activation, and death receptors involving caspase-8 activation. Nuclear accumulation of apoptosis-related factors such as apoptosis-inducing factor (AIF), AIF-homologous mitochondrion-associated inducer of death (AMID), and endonuclease G, on the other hand, trigger caspase-independent apoptosis.

The present study focused upon apoptosis of human malignant pleural mesothelioma (MPM) cells induced by two types of PIs 1,2-dioleoyl-sn-glycero-3-phospho-(1'-myoinositol)(DOPI) and 1,2-dipalmitoyl-sn-glycero-3-phospho-(1'-myo-inositol)(DPPI)(Fig. 1) and aimed at understanding the underlying mechanism. We show here that DOPI and DPPI induce caspase-independent apoptosis of MPM cells by accumulating AIF in the nucleus.

\section{Materials and Methods}

\section{Cell culture}

Human MPM cell lines NCI-H28, NCI-H2052, NCI-H2452, and MSTO-211H cells were purchased from American Type Culture Collection (Manassas, VA, USA). Cells were grown in Roswell Park Memorial Institute (RPMI)-1640 medium supplemented with $10 \%(\mathrm{v} / \mathrm{v}$ ) fetal bovine serum, $0.003 \%(\mathrm{w} / \mathrm{v}$ ) L-glutamine, penicillin $(100 \mathrm{U} / \mathrm{ml})$, and streptomycin $(0.1 \mathrm{mg} / \mathrm{ml})$, in a humidified atmosphere of $5 \% \mathrm{CO}_{2}$ and $95 \%$ air at $37^{\circ} \mathrm{C}$.

Assay of cell viability

Cell viability was evaluated by the method using 3-(4,5-dimethyl-2-thiazolyl)-2,5-diphenyl-2Htetrazolium bromide (MTT).

Terminal deoxynucleotidyl transferase-mediated dUTP nick end labeling (TUNEL) staining

TUNEL staining was performed to detect in situ DNA fragmentation as a marker of apoptosis using an In Situ Apoptosis Detection Kit (Takara Bio, Otsu, Japan). Fixed and permeabilized cells were reacted with terminal deoxynucleotidyl transferase and fluorescein isothiocyanate (FITC)-deoxyuridine triphosphate for 90 min at $37^{\circ} \mathrm{C}$. FITC signals were visualized with a confocal scanning laser microscope (LSM 510, Carl Zeiss Co., Ltd., Oberkochen, Germany). 


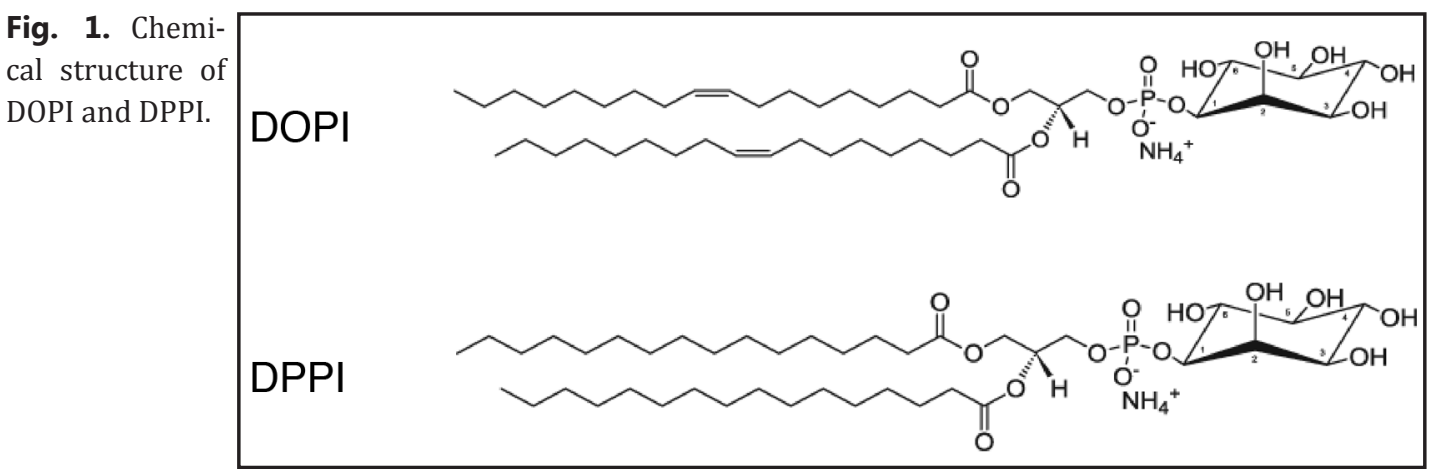

Flow cytometry for apoptosis analysis

Cells were suspended in a binding buffer and stained with both propidium iodide (PI) and annexin V (AV)-FITC, and loaded on a flow cytometer (FACSCalibur, Becton Dickinson, Franklin Lakes, NJ, USA) available for FL1 (AV) and FL2 (PI) bivariate analyisis. Data from 20,000 cells/sample were collected, and the quadrants were set according to the population of viable, unstained cells in untreated samples. CellQuest analysis of the data was used to calculate the percentage of the cells in the respective quadrants.

\section{Monitoring of mitochondrial membrane potentials}

Cells were incubated in a DePsipher ${ }^{\mathrm{TM}}$ solution at $37^{\circ} \mathrm{C}$ for $20 \mathrm{~min}$, and the fluorescent signals were observed with a confocal scanning laser microscope (LSM 510, Carl Zeiss Co., Ltd.) at 543 nm Helium-Neon laser through a long-pass $560 \mathrm{~nm}$ filter for red aggregations and at $488 \mathrm{~nm}$ argon laser through a band-pass 505-530 nm filter for green monomeric form.

Assay of cytochrome c release from the mitochondria

Cells were homogenized with a sonicator in a solution $\left(20 \mathrm{mM}\right.$ HEPES, $10 \mathrm{mM} \mathrm{KCl}, 1.5 \mathrm{mM} \mathrm{MgCl}_{2}$, 1mM EDTA, 1mM EGTA, 1mM dithiothreitol, $0.1 \mathrm{mM}$ phenylmethylsulfonyl fluoride, and $250 \mathrm{mM}$ sucrose, $\mathrm{pH}$ 7.5). Lysates were centrifuged at $1,000 \mathrm{~g}$ for $10 \mathrm{~min}$, and the supernatant was further centrifuged at $10,000 \mathrm{~g}$ for $1 \mathrm{~h}$. The pellet and the supernatant were used as mitochondrial and cytosolic components, respectively. Then, Western blotting was carried out in each component using an anti-cytochrome $\mathrm{c}$ antibody (Chemicon International, Inc., Temecula, CA, USA). Whether the mitochondrial and cytosolic components were successfully separated was confirmed by Western blotting using an anti-VDAC1 antibody (Santa Cruz Biotechnology, Inc., Dallas, TX, USA), a mitochondrial marker.

\section{Enzymatic assay of caspase-3, -4, -8, and -9 activities}

Caspase activity was measured using a caspase fluorometric assay kit (Ac-Asp-Glu-Val-Asp-MCA for a caspase-3 substrate peptide; Ac-Leu-Glu-Val-Asp-AFC for a caspase-4 substrate peptide; Ac-Ile-Glu-Thr-AspMCA for a caspase-8 substrate peptide; and Ac-Leu-Glu-His-Asp-MCA for a caspase- 9 substrate peptide). Cells were harvested and then centrifuged at $800 \mathrm{~g}$ for $5 \mathrm{~min}$ at $4{ }^{\circ} \mathrm{C}$. The pellet was incubated on ice in cell lysis buffer for $10 \mathrm{~min}$ and then, centrifuged at $10,000 \mathrm{~g}$ for $1 \mathrm{~min}$ at $4{ }^{\circ} \mathrm{C}$. The supernatant was reacted with the fluorescently labeled tetrapeptide at $37^{\circ} \mathrm{C}$ for $2 \mathrm{~h}$. The fluorescence was measured at an excitation wavelength of $380 \mathrm{~nm}$ and an emission wavelength of $460 \mathrm{~nm}$ for caspase-3, -8, and -9 or at an excitation wavelength of $400 \mathrm{~nm}$ and an emission wavelength of $505 \mathrm{~nm}$ for caspase- 4 with a fluorescence microplate reader (TECAN Infinite, Männedorf, Switzerland).

Real-time reverse transcription-polymerase chain reaction (RT-PCR)

Total RNAs from cells were purified by an acid/guanidine/thiocyanate/chloroform extraction method using the Sepasol-RNA I Super kit (Nacalai, Kyoto, Japan). RNAs purified were treated with RNase-free DNase I ( 2 units) for $30 \mathrm{~min}$ at $37^{\circ} \mathrm{C}$ to remove genomic DNAs, and $10 \mu \mathrm{g}$ of RNAs was resuspended in water. Then, random primers, dNTP, 10X RT buffer, and Multiscribe Reverse Transcriptase were added to an RNA solution and incubated for $10 \mathrm{~min}$ at $25{ }^{\circ} \mathrm{C}$ and in turn, for $120 \mathrm{~min}$ at $37^{\circ} \mathrm{C}$, to synthesize the first-strand cDNA. Real-time RT-PCR was performed using a SYBR Green Realtime PCR Master Mix (Takara Bio) and the Applied Biosystems 7900 real-time PCR detection system (ABI, Foster City, CA, USA). Thermal cycling 


\section{Cellular Physiology Cell Physiol Biochem 2015;36:1037-1048

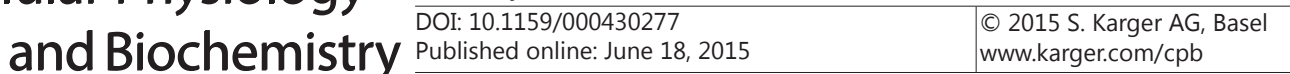 \\ Kanemura et al.: Phosphatidylinositol-Induced Apoptosis}

conditions were as follows: first step, $94{ }^{\circ} \mathrm{C}$ for 4 min; the ensuing 40 cycles, $94{ }^{\circ} \mathrm{C}$ for $1 \mathrm{~s}, 65^{\circ} \mathrm{C}$ for $15 \mathrm{~s}$, and $72{ }^{\circ} \mathrm{C}$ for $30 \mathrm{~s}$. The expression level of each mRNA was normalized by that of GAPDH mRNA. Primers used for real-time RT-PCR are shown in Table 1.

\section{Intracellular distribution of AIF and AMID}

Cells were suspended in a solution [25 mM $\mathrm{MgCl}_{2}, 0.1 \%$ (v/v) Triton X-100, $1 \mathrm{mM}$ dithiothreitol, and $10 \mathrm{mM}$ HEPES, $\mathrm{pH}$ 7.6], and the lysates were centrifuged at $1,100 \mathrm{~g}$ for $5 \mathrm{~min}$ at $4{ }^{\circ} \mathrm{C}$. The pellet and supernatant were used as nuclei- and cytosolenriched components, respectively. Then, Western blotting was carried out in each component using antibodies against AIF (Santa Cruz Biotechnology, Inc., Dallas, Texas, USA) or AMID (GeneTex, Inc., Irvine, CA, USA). Whether the nuclear and cytosolic components were successfully separated was confirmed by Western blotting using an anti-Lamin A/C antibody (Cell Signaling Technology, Inc., Danvers, MA, USA), a nuclear marker.

\section{Nuclear staining}

The nucleus was stained with 4,6-diamidino2-phenylindole (DAPI), and visualized with a confocal scanning laser microscope (LSM 780, Carl Zeiss Co., Ltd., Oberkochen, Germany).
Table 1. Primers used for real-time RT-PCR

\begin{tabular}{lll}
\hline Bad & sense & GCACAGCAACGCAGATGC \\
& anti-sense & AAGTTCCGATCCCACCAGG \\
Bax & sense & CGGACCCGGCGAGAGGC \\
& anti-sense & TCAGCTTCTTGGTGGACGCATCC \\
Bid & sense & CTACGATGAGCTGCAGACTG \\
& anti-sense & GATGCTACGGTCCATGCTGTC \\
Puma & sense & GACGACCTCAACGCACAGTA \\
& anti-sense & AGGAGTCCATGATGAGATTGT \\
Hrk & sense & TGCTCGGCAGGCGGAACTTGTAG \\
& anti-sense & CTTTCTCCAAGGACACAGGG \\
Noxa & sense & GCAGAGCTGGAAGTCGAGTG \\
& anti-sense & GAGCAGAAGAGTTTGGATATCAG \\
Bcl-2 & sense & TCCGCATCAGGAAGGCTAGA \\
& anti-sense & AGGACCAAGGCCTCCAAGCT \\
Bcl-XL & sense & TGGAATTCATGTCTCAGAGCAACCGGGAGC \\
& anti-sense & CAGAATTCTCATTTCCGACTGAAGAGTGAGC \\
Mcl-1 & sense & GGACATCAAAAACGAAGACG \\
& anti-sense & GCAGCTTTCTTGGTTTATGG \\
& anti-sense & AGGTCCACCACCCTGTTGCTGTAG \\
\hline & & \\
\hline
\end{tabular}

Construction and transfection of siRNA

The siRNA silencing the Bid-targeted gene (Bid siRNA) and the negative control siRNA (NC siRNA) were obtained from Ambion (Carlsbad, CA, USA). The sequences of Bid siRNA used here were CUUGCUCCGUGAUGUCUUUtt and AAAGACAUCACGGAGCAAGga. $N C$ siRNA contained the scrambled sequence with the same GC content and nucleic acid composition. Bid siRNA and NC siRNA were reversetransfected into cells using a Lipofectamine reagent (Invitrogen, Carlsbad, CA, USA). Cells were used for experiments $48 \mathrm{~h}$ after transfection.

Statistical analysis

Statistical analysis was carried out using unpaired $t$-test, Dunnett's test, and analysis of variance (ANOVA) followed by a Bonferonni correction.

\section{Results}

DOPI and DPPI induce apoptosis of MPM cells

To see the effect of DOPI and DPPI on MPM cell viability, we initially carried out MTT assay. DOPI or DPPI reduced cell viability for all the investigated MPM cell lines in a concentration (0.01-100 $\mu \mathrm{M}$ )-dependent manner, with a drastic reduction at concentrations more than $10 \mu \mathrm{M}$ (Fig. $2 \mathrm{~A}-\mathrm{D}$ ). There was no difference in the potential between DOPI and DPPI (Fig. 2 A-D).

MPM cells are classified into three types; sarcomatoid, epithelioid, and biphasic tissue types, which include NCI-H28 and NCI-H2052 cell lines, NCI-H2452 cell line, and MSTO$211 \mathrm{H}$ cell line. MSTO-211H cells is relatively rapid as compared with other cell types, and therefore, MSTO- $211 \mathrm{H}$ cells were used for all the ensuing experiments. In the TUNEL staining, KARGER 
Fig. 2. The effects of DOPI and DPPI on MPM cell viability. MTT assay was carried out on NCI-H28 (A), NCI-H2052 (B), NCI-H2452 (C), and MSTO-211H cells (D) treated with DOPI or DPPI at concentrations as indicated for $48 \mathrm{~h}$. In the graphs, each point represents the mean $( \pm$ SEM) percentage of control cell viability (MTT intensities of cells untreated with DOPI or DPPI)(n=4 independent experiments).

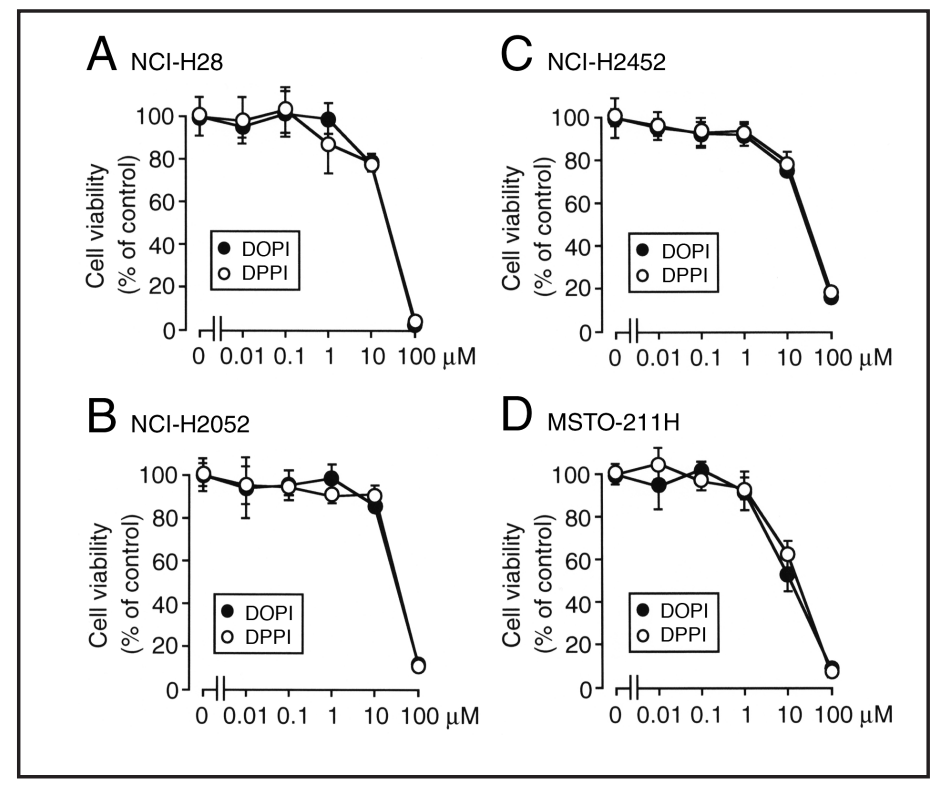

Fig. 3. TUNEL staining. TUNEL staining was carried out on MSTO- $211 \mathrm{H}$ cells treated with DOPI (A) or DPPI (B) at a concentration of 10 $\mu \mathrm{M}$ for $24 \mathrm{~h}$. DIC, Differential interference contrast. Bars $=100 \mu \mathrm{m}$. TUNEL-positive cells were counted in an area $(0.4 \mathrm{~mm} \mathrm{X} 0.4 \mathrm{~mm})$ selected at random. In the graphs, each column represents the mean ( \pm SEM) percentage of TUNEL-positive cells relative to total cells ( $\mathrm{n}=4$ independent experiments). P-Values were defined from unpaired $t$-test.

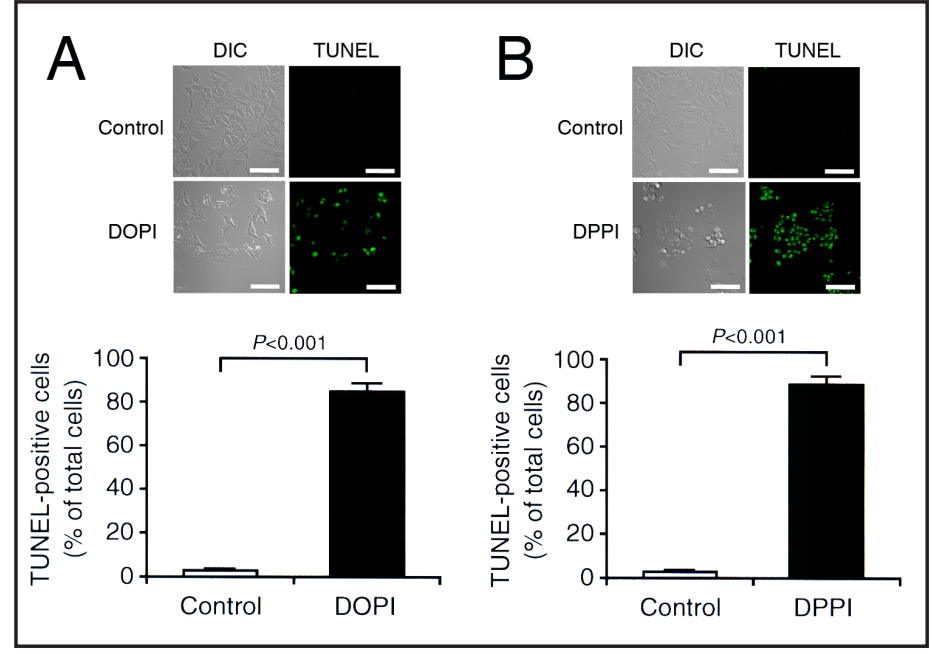

DOPI and DPPI significantly increased TUNEL-positive MSTO-211H cells as compared with untreated cells (Fig. 3 A,B). This indicates that DOPI and DPPI induce apoptosis of MPM cells.

In the flow cytometry using PI and AV, PI is a marker of dead cells and AV, detecting externalized phosphatidylserine residues, is a marker of apoptotic cells [8]. DOPI and DPPI significantly increased the population of PI-positive/AV-negative, PI-negative/AV-positive, and PI-positive/AV-positive MSTO-211H cells, which corresponds to primary necrosis, early apoptosis, and late apoptosis/secondary necrosis, respectively [9], as compared with that for non-treated control cells (Fig. 4). This further supports the notion for DOPI- and DPPIinduced apoptosis of MPM cells. This also suggests that DOPI and DPPI induce necrosis of MPM cells as well.

DOPI and DPPI disrupt mitochondrial membrane potentials but induce caspaseindependent apoptosis of MPM cells

The mitochondria are the major executioner of apoptosis. To see whether DOPI- and DPPI-induced apoptosis of MPM cells is mediated by the mitochondria, we next monitored mitochondrial membrane potentials using DePsipher ${ }^{\mathrm{TM}}$. DePsipher ${ }^{\mathrm{TM}}$, a mitochondrial activity marker, has the properties of aggregating upon membrane polarization forming an orange-red fluorescent compound. If the potential is disturbed, the dye has no access to the transmembrane space and remains in or reverts to its green monomeric form. Only orange- 
Fig. 4. Flow cytometry using PI and AV. MSTO-211H cells were untreated (Control) and treated with DOPI $(10 \mu \mathrm{M})$ or DPPI $(10 \mu \mathrm{M})$ for $24 \mathrm{~h}$. Typical profiles are shown in the upper panel. In the graph, each column represents the mean $( \pm \mathrm{SEM})$ percentage of cell number in 4 fractions against total cell number $(n=4$ independent experi-

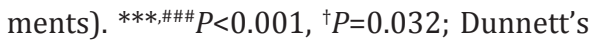
test.

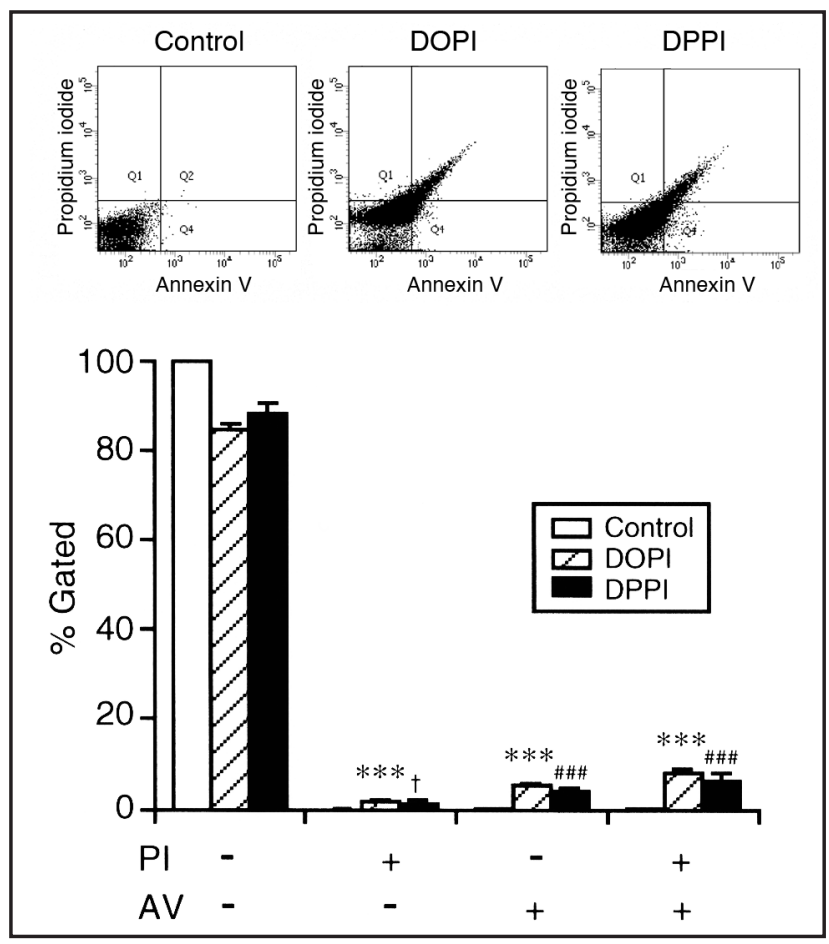

Fig. 5. Monitoring of mitochondrial membrane potentials in MST0-211H cells. Mitochondrial membrane potentials were monitored in cells treated with DOPI $(10 \mu \mathrm{M})$ or DPPI $(10 \mu \mathrm{M})$ for $12 \mathrm{~h}$, and typical images are shown. DIC, differential interference contrast. Bars $=50 \mu \mathrm{m}$. Note that similar results were obtained from 4 independent experiments.

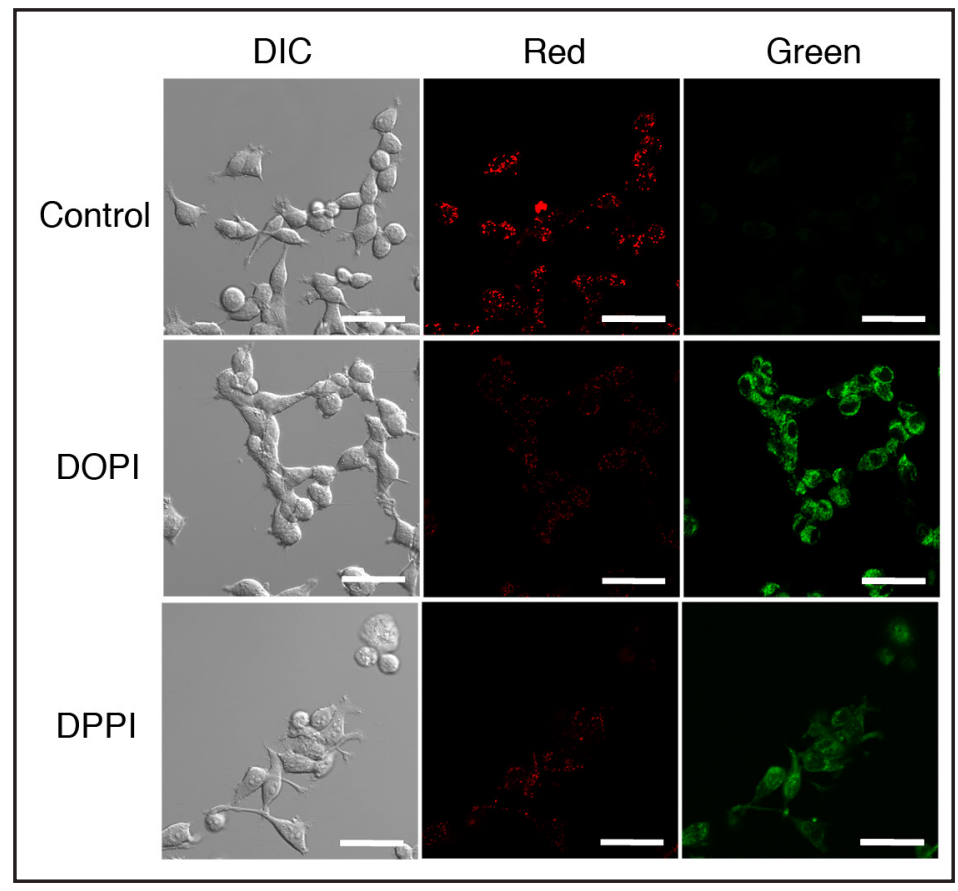

red fluorescent signals were found in non-treated control MSTO-211H cells, but DOPI and DPPI otherwise increased green fluorescent signals in parallel with decreased orangered fluorescent signals (Fig. 5). This indicates that DOPI and DPPI perturb mitochondrial membrane potentials in MSTO-211H cells.

When damaged, the mitochondria release a variety of apoptosis-related factors. We therefore examined intracellular localization of cytochrome c. DOPI increased cytosolic localization of cytochrome $\mathrm{c}$ in MSTO-211H cells at 12-h treatment (Fig. 6A), but no effect was obtained with DPPI (Fig. 6 B). In the enzymatic caspase assay, DOPI and DPPI did not activate caspase- 3 and -9 in MSTO- $211 \mathrm{H}$ cells (Fig. 7 A,B). These results rule out the possibility that DOPI and DPPI disrupt mitochondrial membrane potentials, allowing cytochrome $\mathrm{C}$ 


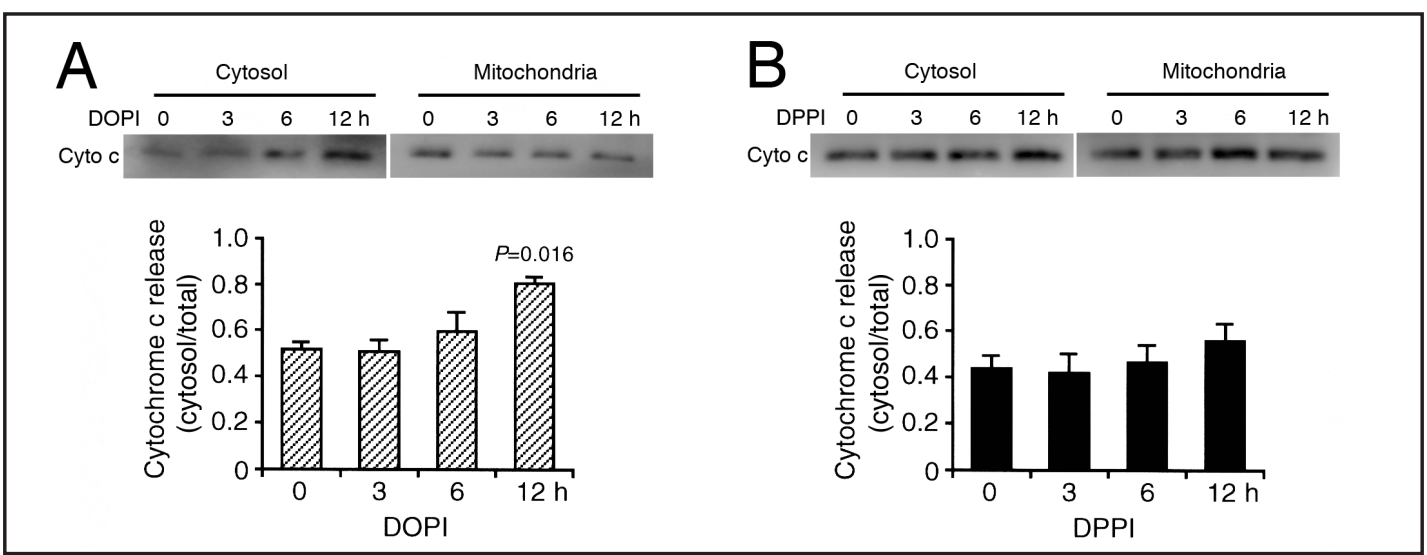

Fig. 6. Cytochrome c release from the mitochondria. After treatment with DOPI (10 $\mu \mathrm{M})$ or DPPI (10 $\mu \mathrm{M})$ for periods of time as indicated, MSTO-211H cells were separated into the cytosolic and mitochondrial components followed by Western blotting. Cyto c, cytochrome c. In the graphs, each column represents the mean $( \pm$ SEM) signal intensity for cytosolic cytochrome $c$ relative to that for whole cell cytochrome $c(n=4$ independent experiments). $P$ value, Dunnett's test.
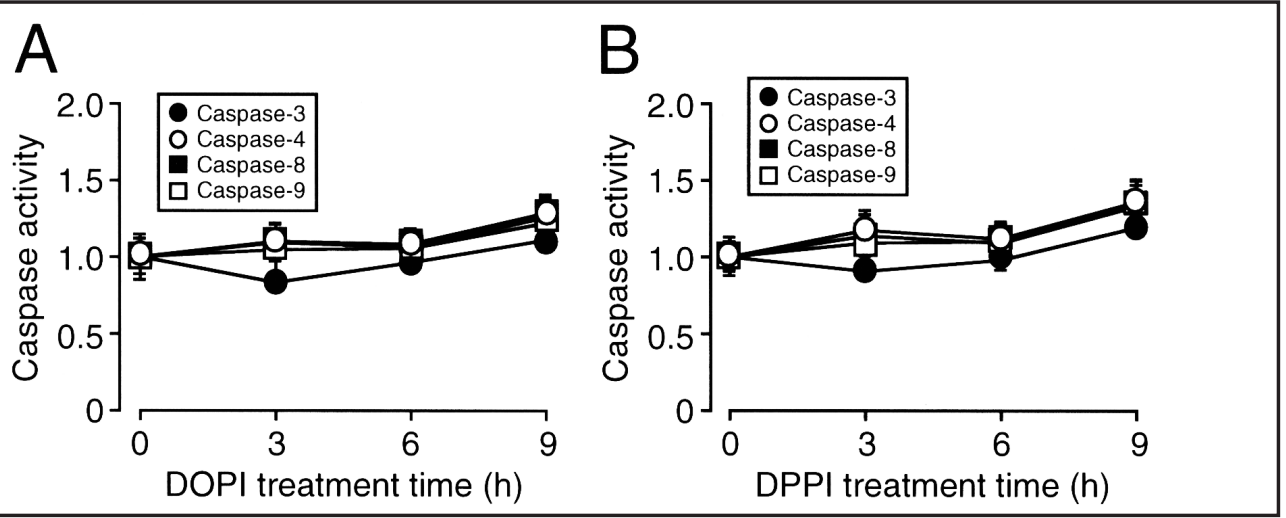

Fig. 7. Assay for caspase-3, -4, -8, and -9 activities. Activities of caspase-3, -4, -8, and -9 were assayed in MSTO-211H cells treated with DOPI (A) and DPPI (B) at a concentration of $10 \mu \mathrm{M}$ for periods of time as indicated. In the graphs, each point represents the mean $( \pm$ SEM) ratio against basal caspase activities $(0 \mathrm{~h})$ ( $n=4$ independent experiments).

release and the ensuing activation of caspase- 9 followed by the effector caspase-3, to induce apoptosis. Moreover, no activation of caspase- 4 and -8 was induced by DOPI and DPPI (Fig. 7 $\mathrm{A}, \mathrm{B})$. Taken together, these results indicate that DOPI and DPPI induce caspase-independent apoptosis of MPM cells.

DOPI and DPPI promote Bid-mediated AIF release from the mitochondria and accumulate AIF in the nucleus of MPM cells

To understand what factors are implicated in DOPI/DPPI-induced apoptosis, realtime RT-PCR was carried out in MSTO- $211 \mathrm{H}$ cells. The Bcl-2 family plays a pivotal role in mitochondrial apoptosis. The BH3-only Bcl-2 family members Bad, Bid, Puma, Hrk, Noxa, Bik, Bim, Blk, and BNIP3 and the Bax subfamily members Bax, Bak, and Bok serve as a proapoptotic factor, but otherwise the Bcl-2 subfamily members Bcl-2, Bcl- $\mathrm{X}_{\mathrm{L}}, \mathrm{Mcl}-1, \mathrm{Bcl}-\mathrm{w}$, and A1 prevents apoptosis.

Both DOPI and DPPI upregulated expression of mRNAs for Bid, but expression of mRNAs for other proapoptotic Bcl-2 family members such as Bad, Bax, Puma, Hrk, and Noxa was not affected (Fig. 8 A,B). This suggests that Bid might participate in DOPI/DPPI-induced apoptosis of MSTO-211H cells.

\section{KARGER}


Fig. 8. Apoptosis-related gene transcription in MSTO-211H cells. Real-time RT-PCR in cells treated with DOPI (A) (C) or DPPI (B)(D) at a concentration of $10 \mu \mathrm{M}$ for periods of time as indicated. The mRNA quantity for each gene was calculated from the standard curve made by amplifying different amount of the GAPDH mRNA, and normalized by regarding the average of independent basal mRNA quantity at $0 \mathrm{~h}$ as 1 . In the graphs, each point represents the mean $( \pm$ SEM) ratio relative to basal mRNA levels $(0 \mathrm{~h})(\mathrm{n}=4$ independent experiments).

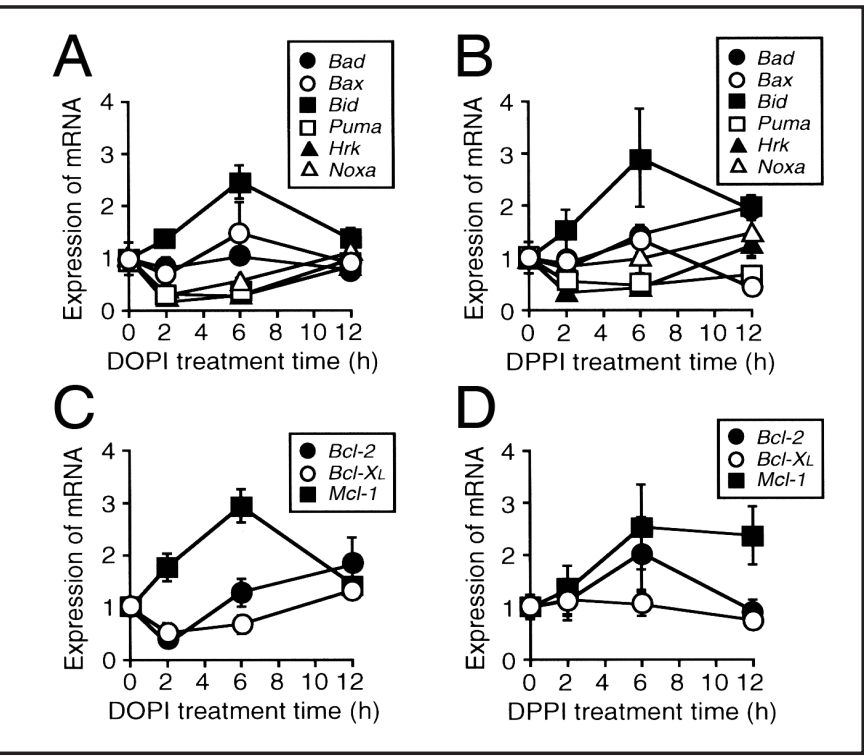

Fig. 9. The effects of DOPI and DPPI on intracellular localization of AIF and AMID. MSTO-211H cells were treated with DOPI (A) or DPPI (B) at a concentration of $10 \mu \mathrm{M}$ for periods of time as indicated, followed by Western blotting. The signal intensity for AIF or AMID protein was normalized by that for $\beta$-actin. In the graphs, each column represents the mean $( \pm$ SEM) normalized intensity for AIF or AMID protein ( $\mathrm{n}=4$ independent experiments). After treatment with DOPI (C) or DPPI (D) at a concentration of $10 \mu \mathrm{M}$ for periods of time as indicated, cells were separated into the cytosolic and nuclear components followed by Western blotting. In the graphs, each column represents the mean $( \pm$ SEM) signal intensity for nuclear AIF or AMID relative to that for whole cell AIF or AMID (n=6 independent experiments). $P$ values as compared with control $(0 \mathrm{~h})$ were defined from Dunnett's test.

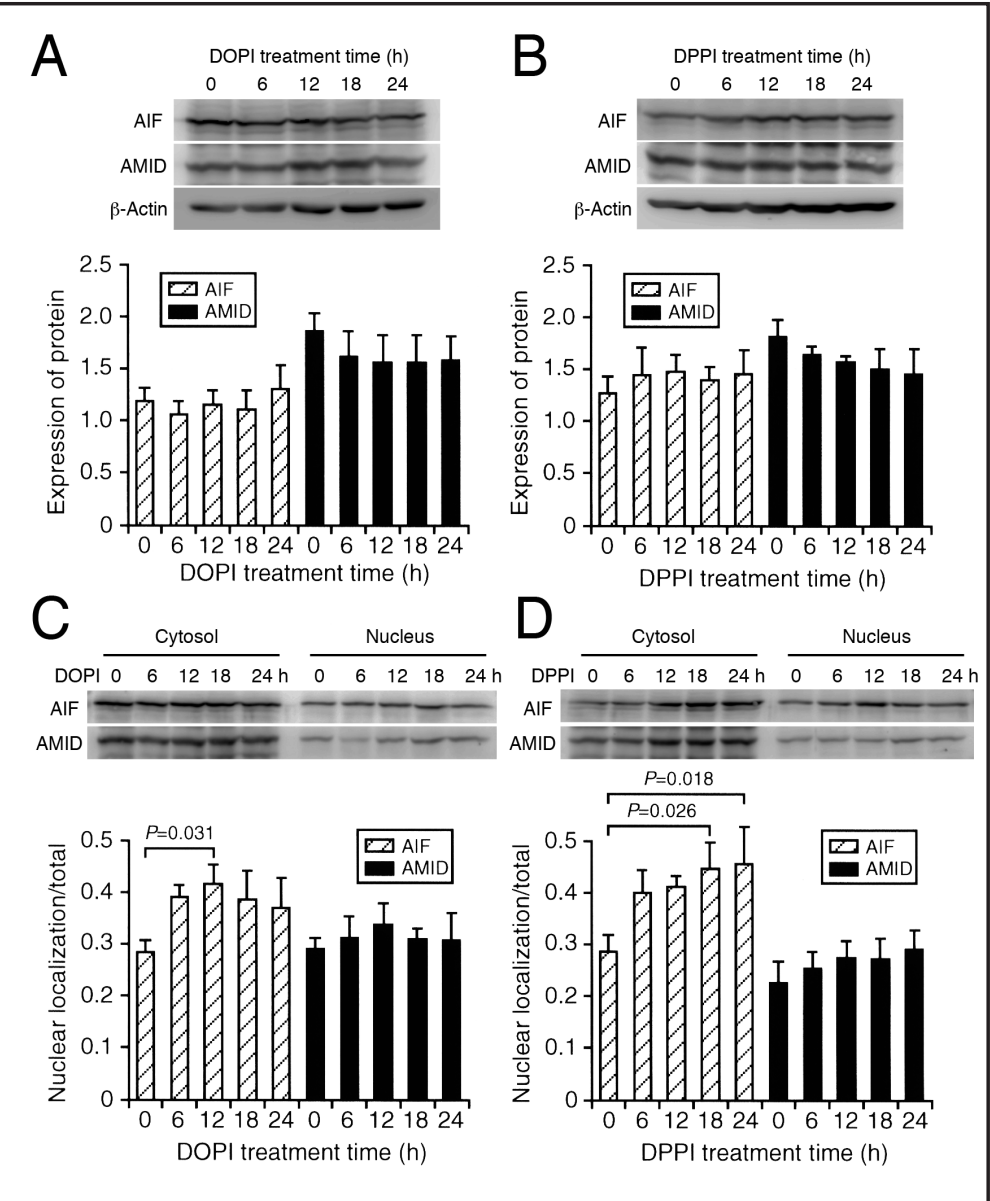

DOPI and DPPI also upregulated expression of the Mcl-1 mRNA, while expression of the mRNAs for other Bcl-2 subfamily members such as Bcl-2 and Bcl- $\mathrm{X}_{\mathrm{L}}$ was not affected (Fig. 8 C,D). Upregulation of Mcl-1, however, does not account for DOPI/DPPI-induced apoptosis of MSTO-211H cells, since Mcl-1 serves as an anti-apoptotic factor.

\section{KARGER}


Fig. 10. DAPI staining. MSTO-211H cells were untreated (Control) and treated with DOPI $(10 \mu \mathrm{M})$ or DPPI $(10 \mu \mathrm{M})$ for $24 \mathrm{~h}$ and then, the nuclei were stained with DAPI. Note that similar results were found with 4 independent experiments. DIC, Differential interference contrast. Bars $=50 \mu \mathrm{m}$.

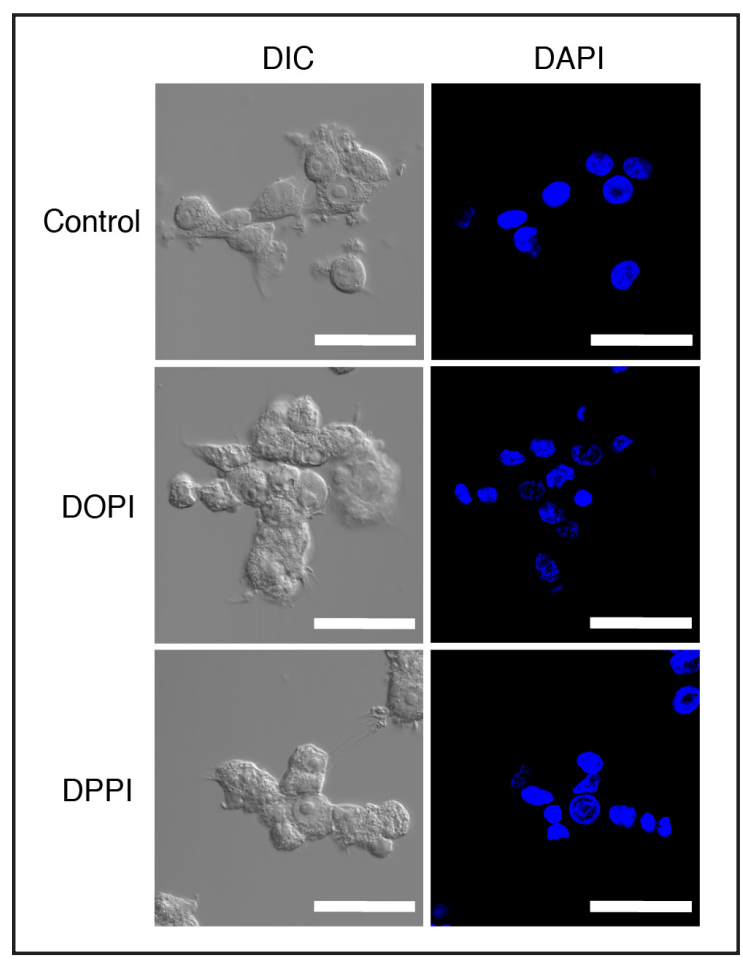

Fig. 11. The role of Bid in DOPI- and DPPI-induced accumulation of AIF in the nucleus. MSTO-211H cells were transfected with siRNAs for NC or Bid, and 48 h later cells were untreated and treated with DOPI (10 $\mu \mathrm{M})(\mathrm{A})$ or DPPI $(10 \mu \mathrm{M})(\mathrm{C})$ for $12 \mathrm{~h}$ followed by Western blotting. In the graphs, each column represents the mean $( \pm$ SEM) signal intensity for Bid normalized by that for $\beta$-actin (n=4-8 independent experiments). $P$ values, unpaired $t$-test. After 12 -h treatment with DOPI $(10 \mu \mathrm{M})(\mathrm{B})$ or DPPI $(10 \mu \mathrm{M})(\mathrm{D})$ in MST0-211H cells transfected with siRNAs for $N C$ or Bid, cells were separated into the nuclear and cytosolic components followed by Western blotting. In the graphs, each column represents the mean $( \pm$ SEM) ratio: signal intensity for nuclear AIF relative to that for whole cell AIF (n=4-8 independent experiments). $P$ values, ANOVA followed by a Bonferonni correction. $N S$, not significant.
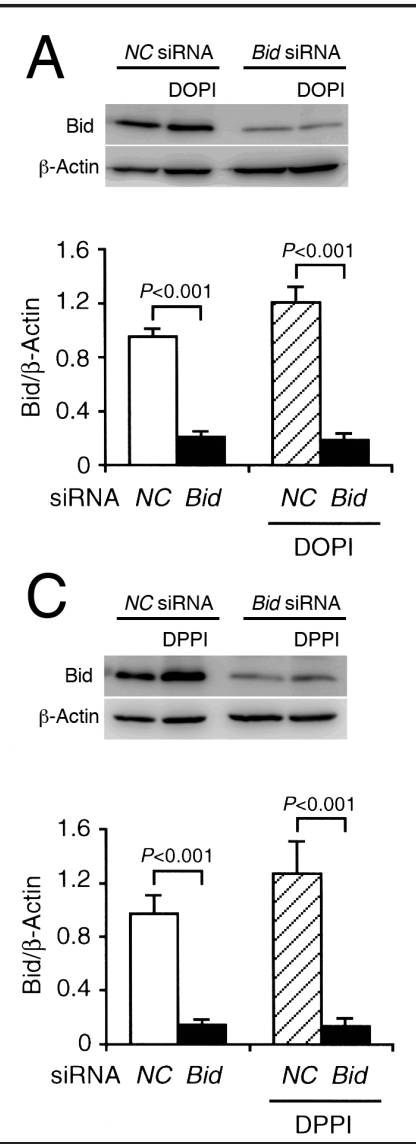
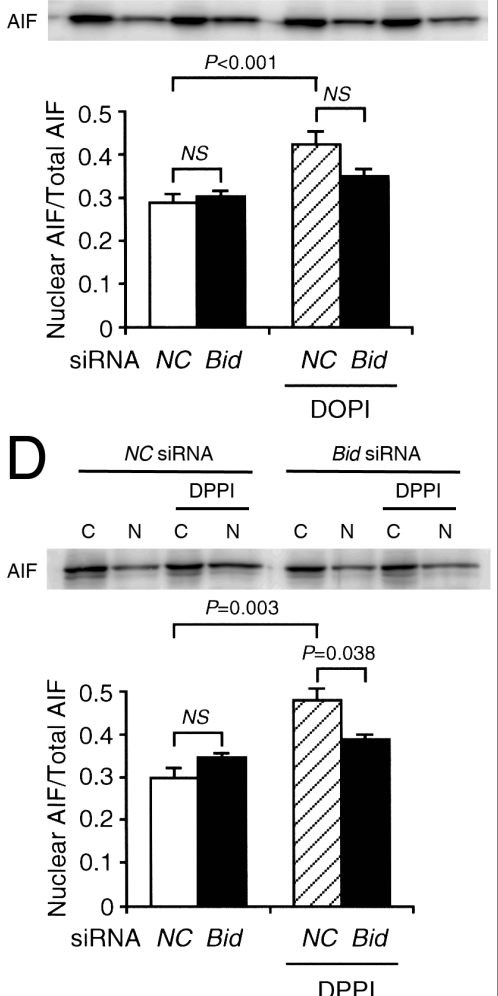

AIF and AMID are released from the damaged mitochondria and accumulate in the nucleus, to induce caspase-independent apoptosis [10-19]. Then, we postulated that AIF and AMID might be implicated in DOPI- and DPPI-induced apoptosis of MPM cells. To address 
Fig. 12. A schematic diagram for DOPI- and DPPI-induced caspase-independent apoptotic pathway.

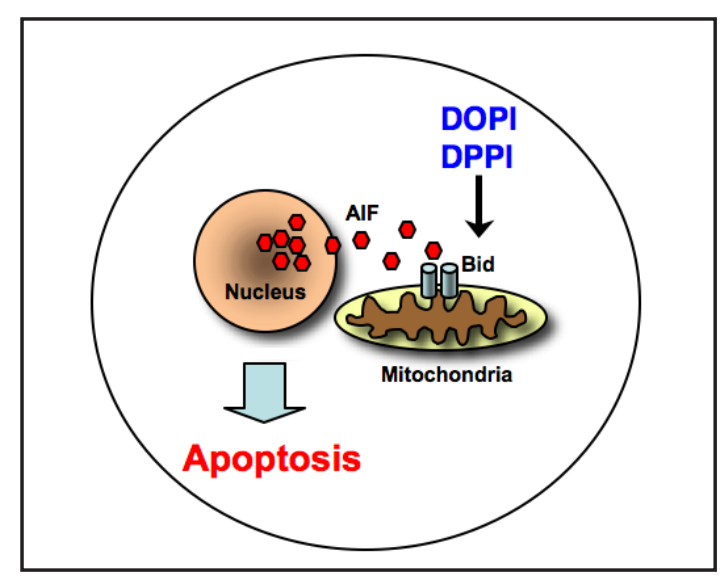

this issue, we examined the effect of DOPI and DPPI on intracellular distribution of AIF and AMID in MSTO-211H cells. DOPI and DPPI had no effect on basal expression levels of AIF and AMID (Fig. 9A,B). In contrast, DOPI and DPPI significantly increased nuclear localization of AIF protein, while intracellular distribution of AMID protein was not affected (Fig. 9 C,D). In the DAPI staining, nuclear fragmentation and condensation were found in cells treated with DOPI and DPPI more obviously than in non-treated control cells (Fig. 10). Collectively, these results indicate that DOPI and DPPI accumulate AIF in the nucleus, to induce caspaseindependent apoptosis of MPM cells.

We finally examined whether Bid is implicated in the nuclear accumulation of AIF induced by DOPI and DPPI. Expression of Bid protein in MSTO-211H cells transfected with the Bid siRNA was clearly suppressed as compared with that in cells transfected with the $N C$ siRNA and this effect was not affected by treatment with DOPI or DPPI (Fig. 11A,C). This confirms that Bid is successfully knocked-down in cells transfected with the Bid siRNA. DOPI and DPPI increased nuclear localization of AIF, which was inhibited by knocking-down Bid (Fig. 11 B,D). Overall, it appears that DOPI and DPPI promotes Bid-mediated AIF release from the mitochondria and accumulate AIF in the nucleus, to induce caspase-independent apoptosis of MPM cells.

\section{Discussion}

AIF as well as AMID are the major executioners for caspase-independent apoptosis [1019]. The mature form of AIF contains three domains: FAD-binding domain, NADH-binding domain, and C-terminal domain. FAD-bound AIF is reduced by NAD(P)H, and reduced AIF catalyzes an NADH-dependent reduction of small molecules such as cytochrome c. The enzymatic function of AIF, however, is not fully understood. The C-terminal domain displays a particular folding consisting of five anti-parallel $\beta$-strands, two $\alpha$-helices and a large loop, exclusive to AIF (amino acids 509-559). This insertion includes a PEST motif followed by a proline-rich module (PPSAPAVPQVP) involved in protein-protein interactions. Notably, the proline-rich domain constitutes a key element in the chromatinolytic/pro-apoptotic AIF function [20].

AIF is imbedded into the inner mitochondrial membrane under the normal conditions. The proapoptotic Bcl-2 family molecules Bax and Bid activated in response to oxidative stress make pores in the mitochondrial membranes. AIF undergoes proteolytic cleavage by cysteine proteases such as calpains and cathepsins [21]. These events allow AIF release from the mitochondria to the cytosol. Once released from the mitochondria to the cytosol AIF translocates to the nucleus in a process positively regulated by cyclophilin A (CypA) and negatively by Hsp70 [22, 23]. On arrival in the nucleus AIF interacts with DNA and/or RNA, to cause chromatinolysis through recruitment of nucleases or to organize a DNA-degrading complex with histone H2AX and CypA, resulting in caspase-independent apoptosis $[10,11]$. 
In the present study, DOPI and DPPI induced apoptosis of MPM cells without activating caspase- $3,-4,-8$, and -9 , while mitochondrial membrane potentials were perturbed. DOPI and DPPI upregulated expression of the Bid mRNA. Moreover, DOPI and DPPI significantly increased nuclear localization of AIF protein and induced nuclear fragmentation and condensation, which were inhibited by knocking-down Bid. Overall, these results indicate that DOPI and DPPI upregulate expression of Bid, to disrupt mitochondrial membrane potentials, allowing AIF release from the mitochondria and AIF accumulation in the nucleus, and then leading to caspase-independent apoptosis of MPM cells (Fig. 12). To our knowledge, this is the first to demonstrate PI signaling related to AIF-mediated apoptosis.

In summary, the results of the present study show that DOPI and DPPI induce caspaseindependent apoptosis of MPM cells by accumulating AIF in the nucleus. This raises the possibility that PI is a target of drug designs for treatment of MPM.

\section{Disclosure Statement}

We have no conflict of interest.

\section{Acknowledgements}

This study was supported by research grants from 'MEXT-Supported Program for the Strategic Research Foundation at Private Universities, 2012-2016'.

\section{References}

1 Nishizuka Y: Intracellular signaling by hydrolysis of phospholipids and activation of protein kinase C. Science 1992;258:607-614.

2 Nishizuka Y: Protein kinase C and lipid signaling for sustained cellular responses. FASEB J 1995;9:484-496.

3 Cantley LC: The phosphoinositide 3-kinase pathway. Science 2002;296:1655-1657.

4 Di Paolo G, De Camilli P: Phosphoinositides in cell regulation and membrane dynamics. Nature Rev 2006;443:651-657.

5 Balla T, Szentpetery Z, Kim YJ: Phosphoinositide signaling: new tools and insights. Physiol 2009;24:231244.

6 Tsuchiya A, Kanno T, Shimizu T, Nakao S, Tanaka A, Nishizaki T: DCP-LA-phosphatidylinositol and its enantiomer exhibit different bioactivities. Cell Physiol Biochem 2014;33:300-309.

7 Tsuchiya A, Kanno T, Shimizu T, Nakao S, Tanaka A, Tabata C, Nakano T, Nishizaki T: A novel PP2A enhancer induces caspase-independent apoptosis of MKN28 gastric cancer cells with high MEK activity. Cancer Lett 2014;347:123-128.

8 Vanags DM, Pörn-Ares MI, Coppola S, Burgess DH, Orrenius S: Protease involvement in fodrin cleavage and phosphatidylserine exposure in apoptosis. J Biol Chem 1996;271:1075-1085.

9 Pietra G, Mortarini R, Parmiani G, Anichini A: Phases of apoptosis of melanoma cells, but not of normal melanocytes, differently affect maturation of myeloid dendritic cells. Cancer Res 2001;61:8218-8226.

10 Yang D, Yaguchi Y, Nagata T, Gotoh A, Dovat S, Song C, Nishizaki T: AMID mediates adenosine-induced caspase-independent HuH-7 cell apoptosis. Cell Physiol Biochem 2011;27:37-44.

11 Cande C, Cecconi F, Dessen P, Kroemer G: Apoptosis-inducing factor (AIF): key to the conserved caspaseindependent pathways of cell death? J Cell Sci2002;115:4727-4734.

12 Daugas E, Susin SA, Zamzami N, Ferri KF, Irinopoulou T, Larochette N, Prévost MC, Leber B, Andrews D, Penninger J, Kroemer G: Mitochondrio-nuclear translocation of AIF in apoptosis and necrosis. FASEB J 2000;14:729-739.

13 Ye H, Cande C, Stephanou NC, Jiang S, Gurbuxani S, Larochette N, Daugas E, Garrido C, Kroemer G, Wu $\mathrm{H}$ : DNA binding is required for the apoptogenic action of apoptosis inducing factor. Nat Struct Biol 2002;9:680-684. 


\section{Cellular Physiology Cell Physiol Biochem 2015;36:1037-1048 \begin{tabular}{ll|l} 
and Biochemistry $\begin{array}{l}\text { DO.1159/000430277 } \\
\text { Published online: June 18, } 2015\end{array}$ & $\begin{array}{l}\text { O 2015 S. Karger AG, Basel } \\
\text { www.karger.com/cpb }\end{array}$ \\
\cline { 2 - 3 }
\end{tabular} \\ Kanemura et al.: Phosphatidylinositol-Induced Apoptosis}

14 Bilyy R, Kit Y, Hellman U, Stoika R: AMID: new insights on its intracellular localization and expression at apoptosis. Apoptosis 2008;13:729-732.

15 Li W, Sun L, Liang Q Wang J, Mo W, Zhou B: Yeast AMID homologue Ndi1p displays respiration-restricted apoptotic activity and is involved in chronological aging. Mol Biol Cell 2006;17:1802-1811.

16 Marshall KR, Gong M, Wodke L, Lamb JH, Jones DJ, Farmer PB, Scrutton NS, Munro AW: The human apoptosis-inducing protein AMID is an oxidoreductase with a modified flavin cofactor and DNA binding activity. J Biol Chem 2005;280:30735-30740.

17 Ohiro Y, Garkavtsev I, Kobayashi S, Sreekumar KR, Nantz R, Higashikubo BT, Usheva A, Gius D, Kley N, Horikoshi N: A novel p53-inducible apoptogenic gene, PRG3, encodes a homologue of the apoptosisinducing factor (AIF). FEBS Lett 2002;524:163-171.

18 Varecha M, Amrichova J, Zimmermann M, Ulman V, Lukasova E, Kozubek M: Bioinformatic and image analyses of the cellular localization of the apoptotic proteins endonuclease G, AIF, and AMID during apoptosis in human cells. Apoptosis 2007;12:1155-1171.

19 Wu M, Xu LG, Li X, Zhai Z, Shu HB: AMID, an apoptosis-inducing factor-homologous mitochondrionassociated protein, induces caspase-independent apoptosis. J Biol Chem 2002;277:25617-25623.

20 Artus C, Boujrad H, Bouharrour A, Brunelle MN, Hoos S, Yuste VJ, Lenormand P, Rousselle JC, Namane A, England P, Lorenzo HK, Susin SA: AIF promotes chromatinolysis and caspase-independent programmed necrosis by interacting with histone H2AX. EMBO J 2010:29:1585-1599.

21 Delavallée L, Cabon L, Galán-Malo P, Lorenzo HK, Susin SA: AIF-mediated caspase-independent necroptosis: a new chance for targeted therapeutics. IUBMB Life 2011;63:221-232.

22 Gurbuxani S, Schmitt E, Cande C, Parcellier A, Hammann A, Daugas E, Kouranti I, Spahr C, Pance A, Kroemer G, Garrido C: Heat shock protein 70 binding inhibits the nuclear import of apoptosis-inducing factor. Oncogene 2003;22:6669-6678.

23 Zhu C, Wang X, Deinum J, Huang Z, Gao J, Modjtahedi N, Neagu MR, Nilsson M, Eriksson PS, Hagberg H, Luban J, Kroemer G, Blomgren K: Cyclophilin A participates in the nuclear translocation of apoptosisinducing factor in neurons after cerebral hypoxia-ischemia. J Exp Med 2007;204:1741-1748. 